\title{
L'impact des réseaux sociaux sur la gestion professionnelle de la communication hospitalière au Royaume-Uni
}

\section{The impact of social media on the professional management of hospital communication in the United Kingdom}

\author{
Pablo Medina* \\ *Faculté de Médecine, Université de Genève, Switzerland \\ Résumé

\begin{abstract}
Le développement des hôpitaux privés, les nouvelles exigences des patients et l'augmentation de l'informations médicale diffusées sur Internet ont poussé certains hôpitaux à parier sur les réseaux sociaux pour ainsi optimiser leurs stratégies de communication institutionnelle et créer des marques réputées. L'objectif de cet article est de savoir si les hôpitaux britanniques considèrent Facebook comme un outil stratégique et utile pour la diffusion de leur marque. Pour ce faire, nous avons réalisé une révision de la littérature sur la communication hospitalière, la marque et les réseaux sociaux ; et, ensuite, nous avons analysé le profil Facebook des 100 meilleurs hôpitaux du Royaume-Uni. La conclusion principale est que, même si la plupart de ces hôpitaux considèrent Facebook comme un outil stratégique, ils n'y diffusent pas tous les éléments composant l'architecture de marque.
\end{abstract}

Mots-clés: hôpital, communication institutionnelle, marque, réputation, réseaux sociaux.

Abstract

The development of private hospitals, the new requirements of patients and the increase of medical information disseminated on Internet have pushed some hospitals to bet on social media in order to optimize their corporate communication strategies and create renowned brands. This paper aims to find out whether British hospitals view Facebook as a strategic and useful tool for their branding actions. To do this, we carried out a literature review about hospital communication, brand and social media; and, then, we analyzed the Facebook profile of top 100 hospitals in United Kingdom. The main conclusion is that, although most hospitals consider Facebook as a strategic tool, they do not disseminate all elements of their brand architecture.

Keywords: hospital, corporate communication, brand, reputation, social media.

\section{Introduction}

Le développement des hôpitaux privés, l'endettement de certains hôpitaux publics, l'augmentation du nombre d'information médicales diffusées sur Internet et les demandes de plus en plus exigeantes de la part des patients ont poussé certains hôpitaux à instaurer une gestion professionnelle de la communication institutionnelle qui leur permette de créer une marque réputée et solide qui soit capable de renforcer les rapports qu'ils établissent avec les stakeholders internes et externes. Pour ce faire, plusieurs hôpitaux parient sur les réseaux sociaux, lesquels sont devenus fondamentaux pour construire des marques solides capables de connecter efficacement avec les stakeholders de l'organisation. L'objectif de cet article est de comprendre comment les hôpitaux britanniques utilisent Facebook pour diffuser les éléments qui composent leur 
architecture de marque (identité, valeurs, mission, vision et culture). Pour ce faire, en premier lieu, nous analysons les concepts de communication institutionnelle, marque, architecture de marque et réseaux sociaux ; et, en deuxième lieu, nous évaluons comment les 100 meilleurs hôpitaux britanniques utilisent Facebook pour diffuser leur architecture de marque.

\section{La communication institutionnelle dans le milieu hospitalier : le défi de la marque $\mathbf{2 . 0}$}

La communication institutionnelle est une activité stratégique de management qui vise la création de rapports de confiance entre l'organisation et ses stakeholders internes et externes (Goodman, 2006). Il s'agit d'une activité transversale qui concerne tous les employés de l'organisation (Illia \& Balmer, 2012) et dont les champs d'activités principaux sont l'identité institutionnelle, l'image, la réputation et les rapports établis entre l'organisation et ses parties prenantes (Frandsen \& Johansen, 2013). La communication institutionnelle apporte à l'organisation une valeur ajoutée qui lui permet d'améliorer ses processus de travail internes et externes ; néanmoins, pour créer cette valeur, il faut considérer la communication d'un point de vue global et multidisciplinaire et inclure dans cette activité le marketing, les relations publiques et la communication interne (Mazzei, 2014). Dans le milieu hospitalier, le développement de la communication institutionnelle fait face à plusieurs contraintes, comme par exemple la difficulté de diffuser des informations médicales et scientifiques, le cadre légal de la santé et de la communication, la confidentialité du patient ou encore les connotations vitales et humaines du service hospitalier proposé au patient. Pourtant, la pression sociale grandissante à laquelle sont soumises toutes les organisations (Christensen \& Cornelissen, 2011) et l'augmentation constante du nombre d'informations médicales diffusées sur Internet (Eysenbach, 2009) obligent les hôpitaux à instaurer une gestion professionnelle de la communication institutionnelle qui leur permette d'établir des rapports de confiance avec leurs stakeholders internes et externes.

Le Département de Communication de l'hôpital assume comme responsabilité principale la construction et diffusion d'une marque solide qui agisse comme lien institutionnel entre l'organisation et toutes ses parties prenantes, notamment les employés et les patients. Selon De Chernatony (2010), la marque est un groupe de valeurs émotionnelles et fonctionnelles qui permet à l'institution d'atteindre ses objectifs organisationnels. Dans le milieu hospitalier, la construction d'une marque capable de rassembler autour d'elle tous les stakeholders est une priorité stratégique parce que ces organisations sont très soumises au changement constant ainsi qu'aux demandes de plus en plus exigeantes de la part des patients (Naveen, Anil \& Smruthi, 2014). Pour construire une marque solide, les hôpitaux peuvent utiliser trois modèles différents: a) le modèle monolithique, dans lequel la marque de l'hôpital est plus importante que celle des départements médicaux de l'organisation; le modèle refrendo, dans lequel la marque de l'hôpital est moins importante que celles des départements médicaux ; ou le modèle mixte, dans lequel la marque de l'hôpital est aussi importante que celle des départements médicaux (Buckley, 2007). La construction d'une marque solide apporte plusieurs avantages à l'hôpital. En premier lieu, la promotion de la marque au niveau interne aide I'organisation à développer une culture positive entre les employés, améliorer leur capacité d'adaptation aux changements et attirer vers l'organisation les meilleurs employés (Drake, Gulman \& Roberts, 2005). En deuxième lieu, la marque aide l'organisation à connecter émotionnellement avec ses clients ainsi qu'à augmenter chez eux l'engagement avec ladite organisation (Kemp, Jillapalli \& Becerra, 2014). Et, en 
troisième lieu, la marque contribue au développement de la réputation de l'organisation, laquelle, dans le cas des hôpitaux, dépend de la confiance qu'ont les patients dans ladite organisation mais aussi des connaissances qu'ils ont sur celle-ci (Hoon Kim et al. 2008).

Pour créer une marque hospitalière solide, le Département de Communication de l'hôpital doit définir et diffuser entre les stakeholders internes et externes les éléments qui composent l'architecture de marque de I'hôpital : identité, valeurs, mission, vision et culture. L'identité fait référence à l'essence d'une organisation et manifeste publiquement sa raison d'être (Nieto, 2005). L'identité institutionnelle constitue une source d'avantage qui permet à l'organisation de fixer une différentiation claire vis-à-vis de ses concurrents. La définition de l'identité est une activité fondamentale pour l'organisation parce que l'identité détermine la stratégie globale de l'organisation ainsi que ses projets sur le moyen et long terme (He \& Balmer, 2008). Une fois définie l'identité, le Département de Communication doit fixer les valeurs institutionnelles de I'hôpital. Les valeurs, non seulement aident I'organisation à prendre des décisions concernant la communication institutionnelle, mais aussi doivent être présents dans tous les comportements des employés ainsi que dans les processus de travail qui ont lieu à l'institution (Jaakson, 2010). Ainsi, il est important que les organisations informent leurs employés sur les valeurs institutionnelles afin que ceux-ci puissent les incorporer à leur logique professionnelle et ainsi diffuser chaque jour, avec leur comportement, l'identité et les valeurs de I'organisation (Aggerholm et al. 2009). Le troisième élément composant l'architecture de marque de l'hôpital est la mission. Selon Nieto (2005), les organisations naissent pour atteindre des objectifs économiques, sociaux et communautaires : l'ensemble de ces objectifs constituent la mission de I'organisation. Au moment de rédiger la mission, les institutions doivent adopter une approche rationnelle parce que les éléments qui composent la mission sont vitaux pour le développement de l'organisation et pour le comportement de ses employés (Verma, 2010). En tenant compte de l'identité, des valeurs et de la mission, le Département de Communication de l'hôpital doit définir la vision de I'organisation. La vision doit décrire l'objectif principal poursuivi par l'organisation et doit être capable, d'une part, d'inclure tous les intérêts de celle-ci et, d'une autre part, de motiver tous les employés, lesquels doivent percevoir la vision comme un élément positif pour l'avenir de l'organisation et de leur propre carrière professionnelle (Kantabutra \& Avery, 2010). La différence principale entre la vision et la mission est que la première est plus axée sur le long terme. C'est pourquoi, elle doit se composer de trois éléments : avenir de l'organisation, objectif de la marque et valeurs institutionnelles (De Chernatony, 2010). Le cinquième et dernier élément qui compose l'architecture de marque est la culture institutionnelle. Ce concept fait référence à la manière unique de travailler qu'ont les employés d'un hôpital et qui les différencient de ses concurrents. Selon Marshal et Adamic (2010), la culture institutionnelle va au-delà du « manuel d'accueil » pour les nouveaux employés : il s'agit d'une narration réalisée par les leaders de l'entreprise qui devient, en partie, une croyance institutionnelle capable de déterminer le comportement de tous les employés. Le vrai objectif de la culture institutionnelle est de pousser les employés à bien faire leur travail, d'où l'importance que les dirigeants de l'organisation réalisent l'importance de cet élément corporatif dans le fonctionnement interne et externe de l'institution (Whiteley, Price \& Palmer, 2013).

La définition de l'architecture de marque (identité, valeurs, mission, vision et culture) et sa diffusion entre les stakeholders internes et externes contribue positivement à la création d'une image de marque positive de l'hôpital. Selon Van Riel (1988), les impressions qu'ont les stakeholders d'une organisation sur les activités de communication massive et interpersonnelle entreprises par ladite organisation donnent lieu à une réalité 
appelée image de marque. Les hôpitaux doivent communiquer d'une manière efficace leur architecture de marque pour que tous les stakeholders aient une image positive sur l'organisation. De cette manière, les hôpitaux commencent à bâtir leur réputation. La gestion efficace de la réputation est une priorité pour le Département de Communication de l'hôpital parce que, selon Van Riel et Fombrun (2007), la réputation apporte une valeur ajoutée à l'institution, à ses activités, à ses employés ainsi qu'à ses produits et ses services.

La diffusion de l'architecture de marque est devenue une activité stratégique pour tous les hôpitaux qui veulent gérer efficacement leur réputation ainsi que les rapports établis avec les stakeholders internes et externes. Et pour ce faire, plusieurs hôpitaux ont recours aux réseaux sociaux. L'interaction proposée par les réseaux sociaux ainsi que la possibilité qu'ils offrent de partager des contenus contribuent à renforcer la vitalité d'une marque ainsi qu'à développer sa valeur ajoutée (Jensen, Muñiz \& Arnold, 2009). Le recours aux réseaux sociaux permet aux organisations de renforcer leur reconnaissance de marque entre les stakeholders ainsi que d'améliorer la loyauté de ces derniers envers l'organisation (Fanion, 2011 ; Erdogmus \& Cicek, 2012). Parmi tous les réseaux sociaux, Facebook est devenu la référence pour toutes les organisations qui veulent réaliser des actions de communication de marque. Il y a cinq raison qui permette de comprendre cette réalité. En premier lieu, le potentiel communicationnel de Facebook (formats, instantanéité, intégration avec d'autres supports, etc.) constitue un avantage pour la création de marques solides et réputées (Linke \&Zerfass, 2013). En deuxième lieu, Facebook propose aux marques une interactivité qui est très utile pour la création de campagnes très efficaces de marketing viral (Thackeray, Hanson \& McKenzie, 2008). En troisième lieu, Facebook constitue une plateforme très utile pour les organisations qui souhaitent développer une communauté de marque (Brodie, et al. 2011). En quatrième lieu, la plupart des consommateurs préfèrent visiter la fan page qu'ont les organisations sur Facebook plutôt que leur site web officiel (Levy \& Birkner, 2011). Et en cinquième lieu, le $80 \%$ des usagers des réseaux sociaux avouent que Facebook est devenu leur outil préféré pour rentrer en contact avec les marques des organisations (Business2Community, 2013). Le Département de Communication de I'hôpital doit adopter une approche professionnelle dans la gestion de Facebook comme un outil de communication institutionnelle capable de contribuer à la création d'une marque hospitalière solide et réputée. Même si certaines organisations diffusent déjà sur Facebook quelques-uns des éléments composant leur architecture de marque, comme par exemple la mission (McCorkindale, 2010), il faut qu'elles adoptent une approche plus professionnelle pour que Facebook devienne un vrai vecteur de communication capable de transmettre aux stakeholders l'identité de l'organisation (Booth \& Matic, 2011).

\section{Méthodologie}

Afin de mieux comprendre le rôle des médias sociaux dans la gestion professionnelle de la communication hospitalière au Royaume-Uni, nous avons analysé le profil Facebook des 100 meilleurs hôpitaux du pays. Pour les choisir, on a eu recours au World Hospitals Ranking élaboré par le Spain's Superior Council of Scientific Research (Consejo Superior de Investigaciones Cientificas de España), I'une des organisations scientifiques les plus réputées en Europe. Pour élaborer le classement des meilleurs hôpitaux britanniques, cette organisation a recours à plusieurs indicateurs lui permettant d'évaluer l'activité professionnelle et de 
recherche de chaque hôpital. Les indicateurs utilisés pour mesurer le facteur d'impact du site internet d'un hôpital sont : visibilité, taille du site, fichiers riches et citations sur Google Scholar. ${ }^{1}$ Afin de comprendre comment les 100 meilleurs hôpitaux britanniques utilisent Facebook pour diffuser leur marque, nous avons analysé leur page officielle sur Facebook, et notamment, trois rubriques : a) a propos, b) évènements et c) vidéos. Pour analyser les trois rubriques, nous avons utilisé 10 indicateurs nous permettant de comprendre le vrai impact de Facebook dans la diffusion des éléments composant l'architecture de marque de l'hôpital (voir table 1). Pour faire cette étude, nous avons considéré uniquement le site officiel de l'hôpital sur Facebook. Ainsi, nous n'avons pas considéré ni les sites des départements ou unités appartenant à l'hôpital ni les sites des groupes globaux auxquels appartiennent certains hôpitaux. ${ }^{2}$

Table 1: Indicateurs analysés sur le profil Facebook

\begin{tabular}{|c|c|c|}
\hline Rubrique & $\begin{array}{l}\text { Éléments Architecture de } \\
\text { Marque }\end{array}$ & Indicateurs \\
\hline \multirow[t]{8}{*}{ A Propos } & \multirow[t]{2}{*}{ Identité corporative } & 1.Description de I’hôpital \\
\hline & & $\begin{array}{l}\text { 2.Liens vers d'autre pages } \\
\text { corporatives }\end{array}$ \\
\hline & Valeurs & $\begin{array}{l}\text { 3.Énumération des valeurs } \\
\text { corporatives }\end{array}$ \\
\hline & Mission & 4.Description de la mission \\
\hline & Vision & 5. Description de la vision \\
\hline & Culture corporative & $\begin{array}{l}\text { 6.Liste des prix reçus par } \\
\text { I'hôpital }\end{array}$ \\
\hline & \multirow[t]{2}{*}{ Image } & $\begin{array}{l}\text { 7.Explication de l'histoire de } \\
\text { I'hôpital }\end{array}$ \\
\hline & & $\begin{array}{l}\text { 8.Présence du logo corporatif } \\
\text { dans l'image principale de la } \\
\text { page Facebook }\end{array}$ \\
\hline Évènements & Image & $\begin{array}{l}\text { 9.Description des évènements } \\
\text { corporatifs }\end{array}$ \\
\hline Vidéos & Culture corporative & $\begin{array}{l}\text { 10. Existence de vidéos où les } \\
\text { protagonistes sont les } \\
\text { professionnels de la santé } \\
\text { travaillant à l'hôpital }\end{array}$ \\
\hline
\end{tabular}

\footnotetext{
${ }^{1}$ Source officielle: http://hospitals.webometrics.info/en/Methodology.

${ }^{2}$ L'analyse a été réalisée du 24 janvier 2017 au 7 février 2017. La liste complète des hôpitaux analysés peut être consultée en annexe 1.
} 


\section{Résultats}

Les réseaux sociaux constituent une opportunité stratégique pour tous les hôpitaux qui souhaitent optimiser leurs efforts de communication institutionnelle et construire une marque réputée capable de générer de la confiance aux différents stakeholders de l'organisation. Les résultats apportés par l'étude réalisée sur l'usage de Facebook par les 100 meilleurs hôpitaux du Royaume-Uni mettent en évidence que certains hôpitaux ne profitent pas encore du potentiel communicationnel de ce réseau social. Néanmoins, la plupart des organisations analysées ont bien compris l'importance de Facebook comme outil de diffusion des éléments composant l'architecture de marque (identité, valeurs, mission, vision et culture). Afin d'expliquer clairement comment les hôpitaux britanniques utilisent ce réseau social pour diffuser leur marque, nous avons divisé cette rubrique de l'article en trois parties : a) analyse des indicateurs, b) architecture de marque et c) modèles à suive.

En premier lieu, l'analyse des indicateurs. La plupart des hôpitaux font confiance à Facebook comme outil de communication institutionnelle. Ainsi, selon les chiffres apportés par cette analyse, le $90 \%$ des hôpitaux considérés ont un profil actif sur Facebook. Néanmoins, le $61 \%$ des hôpitaux respectent uniquement entre 4 et 6 indicateurs (voir Table 2. Nombre d'indicateurs respectés), ce qui met en évidence que les hôpitaux britanniques peuvent encore améliorer la communication réalisée sur ce réseau social. D’ailleurs, il faut noter aussi qu'il y a uniquement un hôpital qui respecte les 10 indicateurs analysés.

En deuxième lieu, l'architecture de marque. Les deux indicateurs les plus respectés sont l'existence de liens vers d'autres sites web corporate de l'hôpital (page web officielle, page web d'un centre de recherche associé à I'hôpital, page web d'un département médical, etc.) et la description de l'organisation ; autrement dit, la plupart des hôpitaux britanniques parient sur la diffusion de leur identité institutionnelle (voir Table 3. Indicateurs les plus respectés). Néanmoins, il y a deux éléments qui font partie de l'architecture de marque de l'hôpital, qui sont très liés à l'identité et qui ne sont pas présents sur le profil Facebook de la plupart des hôpitaux : description des valeurs corporatives $(7,8 \%)$ et de la vision $(4,4 \%)$. Par ailleurs, il faut mettre en avant que le $66,7 \%$ des hôpitaux disposent de vidéos qui portent sur plusieurs sujets -traitements, pathologies, fonctionnement de l'hôpital, évènements, etc.- et sur lesquels les professionnels de la santé travaillant à l'organisation jouent un rôle déterminant. Ceci met en évidence le pari sur ce nouveau langage pour diffuser la marque hospitalière.

En troisième lieu, les modèles à suivre. Selon les résultats apportés par la Table 4. Hôpitaux respectant les plus de critères, il faut signaler le cas de Cumbria Partnership NHS Foundation Trust. Ainsi, il s'agit du seul hôpital respectant les dix indicateurs concernant l'architecture de marque (identité, valeurs, mission, vision, culture et image). Par ailleurs, il y a 4 hôpitaux qui respectent 9 indicateurs et qui méritent aussi être considérés comme des modèles à suivre : Guy's and St Thomas' Hospital NHS, Cambridge University Hospitals NHS Foundation Trust, Sheffield Teaching Hospitals NHS Foundation Trust et University College London Hospitals NHS Foundation Trust. 
Table 2: Nombre d'indicateurs respectés

\begin{tabular}{|l|l|}
\hline Indicateurs & Nombre hôpitaux \\
\hline 10 & 1 \\
\hline 9 & 4 \\
\hline 8 & 4 \\
\hline 7 & 7 \\
\hline 6 & 14 \\
\hline 5 & 23 \\
\hline 4 & 24 \\
\hline 3 & 3 \\
\hline 2 & 5 \\
\hline 1 & 4 \\
\hline 0 & 11 \\
\hline
\end{tabular}

Table 3 : Indicateurs les plus respectés

\begin{tabular}{|l|l|l|}
\hline Indicateurs & $\begin{array}{l}\text { Nombre } \\
\text { hôpitaux }\end{array}$ & Pourcentage \\
\hline 1.Liens d'autres sites corporatifs & 87 & $96,7 \%$ \\
\hline 2.Description de I'hôpital & 84 & $93,3 \%$ \\
\hline 3. Logo comme image de profil & 63 & $70 \%$ \\
\hline 4.Vidéos sur les médecins & 60 & $66,7 \%$ \\
\hline 5.Description évènements & 50 & $55,6 \%$ \\
\hline 6.Explication histoire de l'hôpital & 39 & $43,3 \%$ \\
\hline 7.Description mission & 37 & $41,1 \%$ \\
\hline 8.Liste des prix reçus & 14 & $15,6 \%$ \\
\hline 9.Enumération des valeurs & 7 & $7,8 \%$ \\
\hline 10.Description vision & 4 & $4,4 \%$ \\
\hline
\end{tabular}

Table 4: Hôpitaux respectant le plus de critères

\begin{tabular}{|l|l|}
\hline Hôpital & $\begin{array}{l}\text { Nombre } \\
\text { indicateurs }\end{array}$ \\
\hline 1. Cumbria Partnership NHS Foundation Trust & 10 \\
\hline 2.Guy's and St Thomas' Hospital NHS & 9 \\
\hline $\begin{array}{l}\text { 3.Cambridge University Hospitals NHS Foundation } \\
\text { Trust }\end{array}$ & 9 \\
\hline 4.Sheffield Teaching Hospitals NHS Foundation Trust & 9 \\
\hline $\begin{array}{l}\text { 5.University College London Hospitals NHS } \\
\text { Foundation Trust }\end{array}$ & 9 \\
\hline
\end{tabular}




\begin{tabular}{|l|l|}
\hline 6.Newcastle Upon Tyne Hospitals NHS & 8 \\
\hline 7.NHS Forth Valley & 8 \\
\hline 8.Doncaster and Bassetlaw Hospitals & 8 \\
\hline 9.London Bridge Hospital & 8 \\
\hline
\end{tabular}

\section{Discussion}

L'usage des réseaux sociaux dans le contexte hospitalier constitue un phénomène généralisé et participatif (Weng-Ying et al. 2009). Ces outils s'avèrent être très efficaces pour encourager les gens à s'intéresser au domaine de la santé (traitements, prévention, etc.), mais aussi pour les mettre en contact avec d'autres patients ayant des intérêts similaires (Roblin et al. 2009). Les hôpitaux doivent résoudre les problèmes concernant l'usage des réseaux sociaux (sécurité, confidentialité, gestion de l'identité, etc.) pour ainsi mettre en valeur ces outils comme source d'information médicale à haute valeur ajoutée (Househ, Borycki \& Kushniruk, 2014).

Le $90 \%$ des hôpitaux britanniques ont bien compris l'importance stratégique d'être présent sur Facebook. Néanmoins, il y a très peu d'hôpitaux respectant tous les indicateurs, notamment ceux concernant la liste de prix reçu par l'institution (15,6\%), l'énumération des valeurs (7,8\%) et la description de la vision (4,4\%). Les organisations hospitalières doivent gérer leur présence sur les réseaux sociaux d'une manière plus professionnelle pour ainsi satisfaire les besoins des patients (McCarroll et al. 2014) et attirer le plus grand nombre d'usagers vers leurs profils sur ces réseaux, ce qui contribuera positivement à améliorer la visibilité de leur marque (Huang \& Dunbar, 2013). Les hôpitaux doivent utiliser le potentiel communicationnel de Facebook pour expliquer tous les éléments composant leur architecture de marque. La transmission de ces éléments corporatifs aide les stakeholders à mieux connaitre l'hôpital ; d'où l'importance que ces organisations expliquent, notamment, quelle est leur mission et leur vision. La mission, non seulement influence sur les pratiques professionnelles et la qualité du service proposé par l'organisation (Blair-Loy, Wharton \& Goodstein, 2011), mais aussi aide celle-ci à transmettre aux stakeholders une image univoque et claire (Bartkus \& Glassman, 2008). De son côté, la la vision aide l'organisation à promouvoir la créativité et l'innovation des employés (O'Connell, Hickerson \& Pillutla, 2011), d'où l'importance que cet élément soit défini et disponible sur le profil Facebook.

Selon les résultats apportés par l'étude, le 66,7\% des hôpitaux britanniques ont recours aux vidéos comme outil de diffusion d'informations institutionnelles portant sur les produits et services proposés par l'hôpital ainsi que sur les évènements organisés par celui-ci. L'usage des vidéos pour diffuser des informations médicales est très approprié, notamment quand les patients ont un niveau bas de connaissance sur le sujet traité (Adams, 2010). Le recours au vidéo suppose un défi pour les hôpitaux, mais ça représente aussi une opportunité parce que l'information diffusée sur ce support aide le patient à mieux comprendre certains concepts médicaux ou scientifiques (Gabarrón et al, 2013).

Sur les 90 hôpitaux ayant un profil sur Facebook, 53 respectent au moins cinq indicateurs, ce qui met en évidence que la gestion du profil est assumée par des professionnels de la communication institutionnelle. Les responsables de communication travaillant à I'hôpital doivent analyser les cas de best practices d'autres institutions hospitalières et prioriser toujours une approche compréhensive pour ainsi créer des profils sur 
les réseaux sociaux qui satisfassent vraiment les besoins des patients (Gibbons et al, 2011). Les organisations hospitalières qui gèrent d'une manière efficace leur profil sur Facebook, comme c'est le cas par exemple de Cumbria Parntership NHS Foundation Trust ou Guy's and St Thomas' Hospital NHS, diffusent des informations détaillées et complètes sur leur architecture de marque afin d'établir des rapports de confiance avec les stakeholders et de bâtir ainsi la reputation de l'institution. La présence d'un hôpital sur les réseaux sociaux constitue une vraie opportunité pour la diffusion d'informations crédibles et précises du point de vue scientifique qui aident l'organisation à devenir une source d'information crédible, mais aussi une marque réputée (Lagu et al. 2016 ; Fernandez Silano, 2013).

\section{Conclusion}

Le recours à Facebook comme outil de communication institutionnelle constitue une opportunité pour tous les hôpitaux qui souhaitent établir des rapports de confiance avec leur stakeholders et construire ainsi une marque réputée et crédible. Néanmoins, plusieurs facteurs rendent compliquée cette tâche, comme par exemple la difficulté de divulguer des informations scientifiques ou les contraintes légales et éthiques. Afin de conclure cet article, et en considérant la révision de littérature qui a été réalisée sur la communication et la marque hospitalière à l'ère 2.0, ainsi que les résultats obtenus avec le travail quantitatif mené auprès des 100 meilleurs hôpitaux britanniques, nous pouvons ajouter trois dernières idées. En premier lieu, la plupart des hôpitaux britanniques gèrent Facebook comme un outil stratégique de communication institutionnelle, ce qui est souligné par le fait que le $61 \%$ des hôpitaux respectent entre 4 et 6 indicateurs. En deuxième lieu, la plupart des hôpitaux analysés ne diffusent pas l'ensemble des éléments composant leur architecture de marque ; il faut souligner notamment que la mission n'est diffusée que par le $41,1 \%$ des hôpitaux ; les valeurs, par le 7,8\% des hôpitaux; et la vision, par le 4,4\% des hôpitaux. Les organisations hospitalières devraient diffuser tous les éléments composant l'architecture de marque parce qu'ils sont très liés entre eux et, donc, si l'un d'eux n'est pas diffusé, on ne comprend par la vraie signification de la marque hospitalière. $\mathrm{Et}$, en troisième lieu, la présence non planifiée sur ce réseau social n'apporte rien à I'organisation hospitalière: autrement dit, pour que Facebook devienne un vrai outil stratégique de communication institutionnelle, I'hôpital doit disposer d'une équipe de travail multidisciplinaire -professionnel de la communication, professionnel de la santé, personnel technique, etc.- qui élabore une stratégie de communication online permettant à l'hôpital d'atteindre des objectifs communicationnels précis, quantitatifs et adaptés à la réalité de ce réseau social.

\section{Références bibliographiques}

Adams, S. (2010). Revisiting the online health information reliability debate in the wake of web 2.0: an interdisciplinary literature and website review. International Journal of Medical Informatics, 79 (6), 391-400. Doi: http://dx.doi.org/10.1016/j.ijmedinf.2010.01.006. 
Aggerholm, H., Andersen, M., Asmuß, B. \& Thomsen, C. (2009). Management conversations in Danish companies. Corporate Communications: An International Journal, 14 (3), 264 - 279. Doi: 10.1108/13563280910980050.

Bartkus, B. \& Glassman, M. (2008). Do Firms Practice What They Preach? The Relationship Between Mission Statements and Stakeholder Management. Journal of Business Ethics, 83, (2), 207-216. Doi: 10.1007/s10551-007-9612-0.

Blair-Loy, M., Wharton, A. \& Goodstein, J. (2011). Exploring the relationship between mission statements and work-life practices in organizations. Organization Studies, 32(3), 427-450. Doi: $10.1177 / 0170840610397480$.

Booth, N. \& Matic, Julie (2011). Mapping and leveraging influencers in social media to shape corporate brand perception. Corporate Communications: An International Journal, 16 (3), 184-191. Doi: 10.1108/13563281111156853.

Brodie, R., Ilic, A., Biljana, J. \&Hollebeek, L. (2011). Consumer engagement in a virtual brand community: an exploratory analysis. Journal of Business Research, 61 (1), 105-114. Doi: 10.1016/j.jbusres.2011.07.029

Buckley, P. (2007). The complete guide to hospital marketing. Marblehead: HCPro Inc.

Business2Community (2013). Facebook marketing statistics you need to know. Document retrieved from: http://www.business2community.com/facebook/facebook-marketing-statistics-you-need-to-know0289953.

Christensen, L. \& Cornelissen, J. (2011). Bridging Corporate and Organizational Communication: Review, Development and a Look to the Future. Management Communication Quarterly, 25(3): 383-414. Doi: 10.1007/978-3-531-18961-1_3.

De Chernatony, L. (2010). From brand vision to brand evaluation: the strategic process of growing and strengthening brands. Burlington: Routledge.

Drake, S., Gulman, M. \& Roberts, S. (2005). Light their fire: using internal marketing to ignite employee performance and wow your customers. Chicago: Dearborn Trade Publishing.

Erdogmus, İ. \& Cicek, M. (2012). The impact of social media marketing on brand loyalty. Procedia-Social and Behavioral Sciences, 58, 1353-1360. Doi: 10.1016/j.sbspro.2012.09.1119.

Eysenbach, G. (2009). Infodemiology and infoveillance: framework for an emerging set of public health informatics methods to analyze search, communication and publication behavior on the internet. Journal of Medical Internet Research, 11:e11. Doi: 10.2196/jmir.1157.

Fanion, R. (2011). Social media brings benefits to top companies. Central Penn Business Journal, 27 (3), 76. Doi: 10.1108/MIP-04-2013-0056.

Fernández-Silano, M. (2013). La Salud 2.0 y la atención de la salud en la era digital. Revista Médica de Risaralda, v. 20, n.1, pp. 41-46.

Frandsen, F. \& Johansen, W. (2013). Corporate communication. In V. Bhatia \& S. Bremner, (Eds), The Routledge Handbook of Language and Professional Communication (pp. 220-236). London: Routledge.

Gabarron, E., Fernandez-Luque, L., Armayones, M. \& Lau Annie (2013). Identifying Measures Used for Assessing Quality of YouTube Videos with Patient Health Information: A Review of Current Literature. Interactive Journal of Medical Research, 2(1):e6. DOI:10.2196/ijmr.2465. 
Gibbons, C., Fleisher, L., Slamon, R., Bass, S., Kandadai, V. \& Beck, R. (2011) Exploring the Potential of Web 2.0 to Address Health Disparities, Journal of Health Communication, 16:sup1, 77-89, DOI: 10.1080/10810730.2011.596916.

Goodman, M. (2006). Corporate Communication Practice and Pedagogy at the dawn of New Millenium. Corporate Communication. An International Journal, 11 (3), 196-213. Doi: 10.1108/13563280610680803.

He, H. \& Balmer, J. (2008). Identity studies: multiple perspectives and implications for corporate-level marketing. European Journal of Marketing, 41(7/8), 765-785. Doi: 10.1108/03090560710752393

Hoon Kim, K., Sik Kim, K., Yul Kim, D., Ho Kim, J. \& Hou Kang, S. (2008). Brand equity in hospital marketing. Journal of Business Research, 61 (1), 75-82. Doi: 10.1016/j.jbusres.2006.05.010.

Househ, M., Borycki, E. \& Kushniruk, A. (2014). Empowering patients through social media: The benefits and challenges. Health Informatics Journal, 20 (1), 50-58. Doi: 10.1177/1460458213476969.

Huang, E. \& Dunbar, C. (2013). Connecting to patients via social media: A hype or a reality?. Journal of Medical Marketing: Device, Diagnostic and Pharmaceutical Marketing, 13 (1), 14-23. Doi: http://dx.doi.org/10.1177/1745790413477647

Illia, L. \& Balmer, J. (2012). Corporate communication and corporate marketing. Corporate Communications: An International Journal, 17 (4), 415 - 433. Doi: 10.1108/13563281211274121.

Jaakson, K. (2010). Management by values: are some values better than others? Journal of Management Development, 29 (9), 795 - 806. Doi: 10.1108/02621711011072504

Jensen, H., Muñiz, A. \& Arnould, E. (2009). How brand community practices create value. Journal of Marketing, 73 (5), 30-51. Doi: 10.1509/jmkg.73.5.30.

Kantabutra, S. \& Avery, G. (2010). The power of vision: statements that resonate. Journal of BusinesS Strategy, 31(1), 37-45. Doi: 10.1108/02756661011012769

Kemp, E., Jillapalli, R. \& Becerra, E. (2014). Healthcare branding: developing emotionally based consumer brand relationships. Journal of Services Marketing, 28 (2), 126 - 137. Doi: 10.1108/JSM-08-20120157.

Lagu, T., Goff, S., Craft, B., Calcasola, S., Benjamin, E., Priya, A. \& Lindenauer, P. (2016). Can Social Media Be Used as a Hospital Quality Improvement Tool? Journal of Hospital Medicine, 11 (1), 52-55. DOI: $10.1002 / \mathrm{jhm} .2486$.

Levy, P. \& Birkner, C. (2011). Digital marketing 2011: what you need to know". Marketing News, 45 (3), 1014.

Linke, A. \& Zerfass, A. (2013). Social media governance: regulatory frameworks for successful online communications. Journal of Communication Management, 17 (3), 270 - 286. Doi: 10.1108/JCOM09-2011-0050.

Marshall, J. \& Adamic, M. (2010). The story is the message: shaping corporate culture. Journal of Business Strategy, 31 (2), 18 - 23. Doi: 10.1108/02756661011025035

Mazzei, A. (2014). A multidisciplinary approach for a new understanding of corporate communication. Corporate Communications: An International Journal, 19(2), 216-230. Doi: 10.1108/CCIJ-12-20110073. 
McCarroll, M., Armbruster, S., Chung, J., Kim, J., McKenzie, A. \& Von Gruenigen, V (2014). Health Care and Social Media Platforms in Hospitals. Health Communication, 29 (9), 947-952. DOI: 10.1080/10410236.2013.813831.

McCorkindale, T. (2010). Can you see the writing on my wall? A content analysis of the Fortune 50's Facebook social networking sites. Public Relations Journal, 4 (3), 1-13

Naveen, K., Anil, J. \& Smruthi, T. (2014). Impact of healthcare marketing and branding on hospital services. International Journal of Research Foundation of Hospital \& Healthcare Administration, 2 (1), 1924. Doi: $10.5005 /$ jp-journals-10035-1010.

Nieto, A. (2005). Comunicación institucional: bases para la evaluación. In M.Sevillano (Ed.), Reflexiones en torno a la libertad de empresa informativa(pp. 409-442), Madrid, Spain: Facultad de Ciencias de la Información, Universidad Complutense de Madrid.

O'Connell, D., Hickerson, K. \& Pillutla, A. (2011) Organizational Visioning: An Integrative Review. Group \& Organization Management, 36, 103-25. Doi: 10.1177/1059601110390999.

Roblin, D., Houston, T., Allison, J., Joski, P. \& Becker, E. (2009). Disparities in use of a personal health record in a managed care organization. Journal of the American Medical Informatics Association, 16, 683-689. Doi: 10.1197/jamia.M3169.

Thackeray, R., Hanson, C. \& McKenzie, J. (2008). Enhancing Promotional Strategies Within Social Marketing Programs: Use of Web 2.0 Social Media. Health Promotion Practice, 9 (4), 338-43. DOI: $10.1177 / 1524839908325335$.

Van Riel, C. (1998). Comunicación corporativa (Translate E. Cerdá). Madrid: Prentice Hall.

Van Riel, C. \& Fombrun, C. (2007). Essentials of corporate communication: implementing practices for effective reputation gestion. Abingdon: Routledge.

Verma, H. (2010). Mission statements-a study of intent and influence. Journal of Services Research, 9, (2), 153-172.

Weng - Ying, C., Hunt, Y., Beckjord, E., Moser, R. \& Hesse, B. (2009). Social media use in the United States: implications for health communication. Journal of Medical Internet Research, 11(4), e48. Doi:10.2196/jmir.1249.

Whiteley, A., Price, C. \& Palmer, R (2013). Corporate culture change: adaptive culture structuration and negotiated practice. Journal of Workplace Learning, 25 (7), 476 - 498. Doi: 10.1108/JWL-09-20120069.

\section{Annexes}

\section{Annexe 1. Liste complète des hôpitaux analysés :}

1)Royal Berkshire NHS Foundation Trust; 2) Guy's and St Thomas' Hospital NHS; 3) NHS Greater Glasgow and Clyde; 4) Institute of Cancer Research Royal Cancer Hospital; 5) NHS Fife; 6) BMI Health Care; 7) Royal Marsden Hospital; 8) Nuffield Health Hospitals; 9) Cambridge University Hospitals NHS Foundation Trust; 
10) North Bristol NHS; 11) London Fertility Centre; 12) Royal United Hospital NHS Trust; 13) NHS Lothian; 14) Central Manchester University Hospitals NHS Foundation Trust; 15) Merseycare NHS; 16) NHS Wirral Health Service; 17) University Hospital Southampton NHS; 18) South London and Maudsley NHS Foundation Trust; 19) Queen Elizabeth Hospital King's Lynn NHS Trust; 20) Nottingham University Hospitals NHS Trust; 21) NHS Grampian; 22) Royal Cornwall Hospitals Trust; 23) Leicester's Hospitals; 24) Cumbria Partnership NHS Foundation Trust; 25) Papworth Hospital NHS Foundation Trust; 26) Oxford University Hospitals NHS Trust; 27) Wrightington Wigan and Leigh NHS Trust; 28) North East London Mental Health NHS Trust; 29) Great Ormond Street Hospital; 30) Heart of England NHS Foundation Trust; 31) University Hospitals Bristol NHS Foundation Trust; 32) Christie Hospital NHS; 33) Northumberland, Tyne and Wear NHS Foundation Trust; 34) Sheffield Teaching Hospitals NHS Foundation Trust; 35) Newcastle Upon Tyne Hospitals NHS; 36) Royal Liverpool University Hospital; 37) University Hospital Birmingham NHS; 38) Leeds Teaching Hospitals NHS Trust; 39) Chelsea and Westminster Hospital; 40) University College London Hospitals NHS Foundation Trust; 41) Sandwell And West Birmingham Hospital Swbh NHS; 42) Shrewsbury \& Telford Hospital NHS Trust; 43) King's College Hospital NHS Foundation Trust; 44) Salisbury NHS Foundation Trust; 45) Norfolk and Norwich University Hospital NHS; 46) West Hertfordshire Hospitals; 47) Northern Devon Healthcare Trust; 48) Brighton and Sussex University Hospitals NHS Trust; 49) NHS Forth Valley; 50) Plymouth Hospitals NHS Trust; 51) NHS Tayside; 52) Gloucestershire Hospitals NHS Foundation Trust; 53) University Hospital of South Manchester; 54) Doncaster and Bassetlaw Hospitals; 55) Homerton University Hospital; 56) Royal Bournemouth and Christchurch Hospitals NHS Foundation Trust; 57) South West Yorkshire Partnership NHS Foundation Trust; 58) St George's Healthcare Trust; 59) London Bridge Hospital; 60) Moorfields Eye Hospital NHS Foundation Trust; 61) Northern Health and Social Care Trust; 62) Lancashire Care NHS Foundation Trust; 63) South West London and St George's Mental Health NHS Trust; 64) Old Harlow Health Centre Jenner House; 65) Royal National Orthopaedic Hospital NHS Trust; 66) Priory Group; 67) Birmingham Children's Hospital NHS Trust; 68) Royal Devon and Exeter NHS Foundation Trust; 69) East \& North Hertfordshire NHS Trust; 70) Sherwood Forest Hospitals NHS Foundation Trust; 71) Robin Lane Medical Centre NHS; 72) St Helens \& Knowsley NHS Trust; 73) Gleadless Medical Centre; 74) Bedford Hospital NHS Trust; 75) Portsmouth Hospitals NHS Trust; 76) Central and North West London Mental Health Trust; 77) West London Mental Health NHS Trust; 78) NHS Ayrshire \& Arran; 79) Surrey and Sussex Healthcare NHS Trust; 80) Health and Social Services Board; 81) West Suffolk Hospital NHS; 82) South Tees Hospitals NHS Trust; 83) Leicestershire Partnership NHS Trust; 84) United Lincolnshire Hospitals NHS; 85) Oxleas NHS Foundation Trust; 86) Northampton General Hospital; 87) Hertfordshire Community NHS Trust; 88) Clatterbridge Centre for Oncology NHS Foundation Trust; 89) Sussex Partnership NHS Foundation Trust; 90) Surrey and Borders Partnership NHS Foundation Trust; 91) Tees Esk and Wear Valleys NHS Trust; 92) Avon and Wiltshire Mental Health Partnership NHS Trust; 93) Maidstone and Tunbridge Wells NHS Trust; 94) Calderdale \& Huddersfield NHS Foundation Trust; 95) Christ's Hospital; 96) Hampshire Hospitals NHS Foundation Trust; 97) NHS Orkney; 98) Buckinghamshire Hospitals NHS Trust; 99) British Institute of Radiology; et 100) Torbay Care Trust. 